\title{
ACTITUDES, CONOCIMIENTOS Y PRÁCTICAS DE MADRES DE NIÑOS EN EDAD PREESCOLAR FRENTE A LA ALIMENTACIÓN DE SUS HIJOS, UN ACERCAMIENTO A LA REALIDAD DE LA COMUNA 9 DE ARMENIA (QUINDÍO)
}

\author{
KNOWLEDGE, ATTITUDES AND PRACTICES ANALYSIS OF ALIMENTARY \\ PRACTICES FROM MOTHERS OF PRESCHOOL CHILDREN IN THE URBAN \\ COMMUNITY 9 OF ARMENIA (QUINDIO)
}

\begin{abstract}
Sebastian Arias Pérez ${ }^{1 *}$, Ángela María Ramírez Soler ${ }^{1}$, Jennifer Paola Valencia Burbano ${ }^{1}$, Leidy Geraldine Villa Encizo ${ }^{1}$, Diana Patricia Londoño Buriticá ${ }^{2}$, Cesar Augusto Gómez Santos ${ }^{3}$.
\end{abstract}

1. Programa de Enfermería. Corporación Universitaria Empresarial Alexander von Humboldt.

2. Programa de Enfermería. Corporación Universitaria Empresarial Alexander von Humboldt. email: dianlondo59@cue.edu.co

3. Programa de enfermería, Corporación Universitaria Empresarial Alexander von Humboldt.email: cgomez@cue.edu.co *Correspondencia del Autor: Leidy Geraldine Villa Encizo. Mz 42 casa 1 barrio La Grecia
e- mails: Ladyg9408@gmail.com - lvilla36@cue.edu.co

Cómo citar:

Arias et al. (2017) ACTITUDES, CONOCIMIENTOS Y PRÁCTICAS DE MADRES DE NIÑOS EN EDAD PREESCOLAR FRENTE A LA ALIMENTACIÓN DE SUS HIJOS, UN ACERCAMIENTO A LA REALIDAD DE LA COMUNA 9 DE ARMENIA (QUINDÍO). Revista de Investigaciones de la Universidad del Quindío. Vol 29, pp.16-27.

Información del artículo: Recibido: 26 noviembre 2016; Aceptado: 16 mayo 2017 


\title{
RESUMEN
}

Los niños requieren cuidado permanente. A medida que éstos van creciendo, adquieren las bases para generar sus propios hábitos saludables. Generalmente la madre como cuidadora principal, es quien se encarga de fortalecer y afianzar en sus hijos, aspectos relacionados con la forma adecuada de alimentarse.

El presente documento da cuenta de los resultados de una investigación cuantitativa de corte transversal, que tuvo como objetivo la descripción de los conocimientos, las actitudes y las prácticas de las madres frente a la alimentación de sus hijos en edad preescolar, en la comuna 9 de la ciudad de Armenia, Quindío. Utilizó un muestreo intencional y un instrumento de recolección de información tipo CAP (Conocimientos, Actitudes y Prácticas) diseñado por los autores y aplicado a 50 mujeres con edades que oscilaron entre los 18 y 54 años, previa aplicación de prueba piloto y firma del consentimiento informado por parte de los participantes. El análisis estadístico se realizó mediante la utilización del programa Excel para procesar datos, versión 2010 de Microsoft. Se aplicó estadística descriptiva a los datos recabados.

Dentro de los principales hallazgos se evidenció que las madres reconocen el concepto de alimentación saludable y logran identificar los grupos de alimentos más importantes en la dieta de sus hijos. Frente a las actitudes, las mujeres encuestadas se muestran indiferentes y evidencian prácticas alimentarias deficientes.

La literatura actual sobre la temática, es limitada; sin embargo, los hallazgos del presente estudio, se contrastaron con reportes de investigaciones nacionales e internacionales.

Palabras claves: conducta alimentaria, madres, preescolares, encuestas nutricionales, conductas saludables.

\begin{abstract}
Children require permanent care as they grow, they acquire the bases to generate their own healthy habits. Usually is the mother, as the primary caregiver, the person that have the responsibility of strengthen in their children the aspects related with the correct way of feeding
\end{abstract}

This paper reports the results of a quantitative cross sectional research, which aimed at the description of the knowledge, attitudes and practices of mothers about feeding their preschool children in the district 9 the city of Armenia, Quindío. It was applied a non- Probabilistic sample. A self-administered questionnaire type CAP, (Knowledge, Attitudes and Practices), was applied to fifty women between aged eighteen and fifty four years of age, which was designed by the authors, a pilot test was applied to the questionnaire. Statistical analysis was performed in Microsoft Excel version 2010.

An important finding in this study is that mothers recognize healthy food, additionally they can identify the most important food's groups in the diet of their children. Women that answered the questionnaire show indifference in relation with the attitudes about the feeding, they have poor feeding practices too.

The current literature on the subject is limited; however, the findings of this study were contrasted with reports from national and international research

Keywords: feeding behavior, mothers, preschool child, nutrition survey, health behavior. 


\section{INTRODUCCIÓN}

Según Franco, “La alimentación es una construcción social y cultural. Los alimentos para ser susceptibles de consumo, pasan por un proceso de transformación que expresa normas culturales de clasificación y combinación1" (Franco, 2010, p.2). Desde esta perspectiva, en palabras de Osses, S, Macías, C, Castaño, S, López, A y Gómez del Castillo, D. (2014)2, la alimentación se concibe como una construcción que trasciende las dinámicas puramente prácticas. En la primera infancia, la alimentación constituye una práctica privilegiada en la que se conjugan posibilidades de aprendizaje, intercambio social, construcción de autonomía, participación y creación de vínculos, así como la apropiación de destrezas y habilidades clave en el desarrollo integral de los niños.

La alimentación, y concretamente la alimentación en la infancia como objeto de estudio, se ha orientado desde enfoques disciplinares que profundizan en problemáticas concretas. Así, desde las ciencias sociales se han enfocado los estudios a las dimensiones de desigualdad, pobreza y seguridad alimentaria; a aspectos históricos vinculados al ámbito de la construcción de valores culturales en procesos identitarios y a aspectos culturales principalmente en comunidades originarias o minoritarias. En las ciencias de la salud, predominan estudios sobre los trastornos alimenticios, el aspecto nutricional y la asociación de determinados consumos alimentarios con enfermedades particulares.

Los niños al nacer deben tener como referente una persona que esté a cargo de sus cuidados. A medida que el niño va creciendo, adquiere las bases para generar sus propios hábitos y si la información y formación recibida es positiva, ésta permitirá que los chicos opten por prácticas saludables; en este caso y específicamente prácticas saludables alrededor de la alimentación3-4.

Desde el nacimiento hasta la muerte la persona está en una constante necesidad de aprender cosas nuevas, ya que éstas son las que le ayudarán a generar el proceso de adaptación al mundo y al entorno en el que se desenvuelve cotidianamente. En las primeras etapas de la vida el aprendizaje se da de manera rápida, ya que el menor aprende de una forma visual y repetitiva, es por esto que acostumbra a imitar muchos de los actos de las personas a su alrededor, por eso es muy importante un acompañamiento por parte de los padres para que los actos que éstos realicen, sean bien replicados por los niños.

La madre es el referente familiar más cercano de toda persona, ya que el lazo que se genera entre una madre y un hijo es muy fuerte, de esta manera la madre es quien le brinda a su hijo el primer aprendizaje, le enseña desde cómo moverse, vestirse, hasta como alimentarse. En relación a éste último aspecto, lo primero que se le ofrece al recién nacido es la leche materna y gradualmente, se van mostrando e incorporando variedad de alimentos que el niño necesita para su crecimiento y desarrollo. Según Barrera (2014) "En la edad preescolar es donde el menor tomará esos conocimientos de manera positiva y los adoptará según su avance en el ciclo vital"5.

Una apropiada alimentación según la edad, proveerá los nutrientes esenciales e indispensables para el adecuado crecimiento, desarrollo y mantenimiento de la salud de las personas. Cuando un niño no recibe los nutrientes necesarios para su desarrollo y padece de malnutrición crónica, se presenta retraso en el crecimiento, se afecta el sistema inmunitario, se disminuye el desarrollo cerebral y su capacidad de aprendizaje. En los adolescentes los problemas nutricionales comienzan con la niñez y continúan durante la vida adulta6.

La malnutrición se asocia a la pobreza, al alza en los precios de los alimentos, a la escases de los mismos en zonas de conflicto y a las catástrofes naturales que reducen el acceso de las familias a los alimentos apropiados y en cantidad suficiente6.

La malnutrición es uno de los factores que más contribuye a la carga mundial de morbilidad. Más de una tercera parte de las defunciones infantiles en todo el mundo se atribuye a la desnutrición (alrededor de 1,5 millones al año), especialmente en África y Asía. Según reportes del año 2011, en África afecta al 42\% de la población infantil6.

Según la Organización Mundial de la Salud, en el año 2010 , cerca de 40 millones de niños y niñas menores de 5 años de edad presentaban sobrepeso y obesidad a nivel mundial (Organización Mundial de la Salud, 2010)7, si se compara con otros años estas cifras se han incrementado, según un reporte del Banco Mundial en el año 2012, existían 44 millones de niños(as) afectados con este problema (UNICEF/WHO/World Bank, 2013)8. 
La situación de los niños menores de 5 años en el mundo y en particular en América Latina requiere atención especial, se observa elevados índices de enfermedades prevalentes como problemas respiratorios agudos, diarreas y desnutrición, los cuales son prevenibles con prácticas saludables de bajo costo y de fácil aplicación.

Frente a esta situación, es urgente realizar esfuerzos en la labor de afrontar con seriedad este problema; la adopción de prácticas de alimentación adecuadas durante los primeros cinco años de vida (incluyendo la lactancia materna exclusiva los primeros 6 meses, así como la preparación para el destete, la continuación de la lactancia en forma complementaria hasta los 2 años, la detección precoz del retraso del crecimiento y desnutrición, entre otros), disminuirían los índices de enfermedades prevalentes en la infancia.

A pesar de que mediante intervenciones de bajo costo y alta efectividad como las citadas, la realidad en Colombia es bien distinta:

Según la Encuesta Nacional de Demografía y Salud ENDS (2010)9, se demostró que la lactancia materna exclusiva tiene muy corta duración con una mediana de 1,8 meses. Otro estudio realizado por Sáenz M (2007)10, muestra que en Colombia el 83,8\% de las madres inician la alimentación complementaria antes del cuarto mes de vida, prácticas que pueden ser lesivas para el menor de cinco años.

Según los indicadores básicos, la desnutrición crónica en menores de 5 años fue del 13,2\% y la desnutrición aguda del 0,90\% para el año 20105.

Parada Rico (2011)11, encontró que en Colombia existen determinantes de riesgo en grupos de la población, tales como la ausencia de conocimientos acertados sobre la alimentación complementaria y micronutrientes, lo cual genera prácticas inadecuadas derivadas de creencias en torno a la alimentación, las cuales confluyen en el inicio temprano de alimentación complementaria, según sus cuidadores, con el objetivo de que el menor al probar los alimentos que consume la familia "se vuelva más fuerte a las infecciones o se les cure el estómago" o por el contrario el inicio en forma tardía de la misma, todo lo anterior enmarcado en un ciclo de creencias que han adoptado los cuidadores desde sus ancestros.
Durante el periodo 2005-2010 en el departamento del Quindío, la prevalencia de desnutrición crónica en menores de 5 años aumentó de $12 \%$ a $13 \%$. La tasa de mortalidad por desnutrición para la misma población, durante el año 2013 fue de 2,17/100.000 niños menores de 5 años, 2 puntos por debajo que la tasa nacional. Del mismo modo, la desnutrición se encontró dentro de las primeras 5 causas de consulta en menores de 5 años, con mayor proporción en el grupo de mujeres13.

En este estudio se tomó como referencia, las madres de niños en edad preescolar (entre los 3-5 años de edad), edad en la cual se carece de habilidades para el autocuidado y de la autonomía necesaria para la toma de decisiones. Sin embargo, es una edad en la cual el aprendizaje se da de forma rápida, de tal manera que lo que los niños aprenden y experimentan se refleja en los hábitos que lo acompañarán por el resto de su vida.

El "plato saludable" es una herramienta desarrollada por el Ministerio de Salud y Protección Social y el Instituto Colombiano de Bienestar Familiar (ICBF) como referente para los colombianos en la elección de los alimentos, distribuidos en seis grupos y las porciones diarias de los mismos, que aporten el contenido calórico y de nutrientes necesarios e indispensables para su sano y correcto desarrollo. Las recomendaciones indican horarios de comidas (desayuno, almuerzo y comida), e invita a consumir agua y a realizar actividad física de forma regular. Los grupos de alimentos incluidos en el plato saludable son: 1 . cereales, tubérculos, raíces y plátanos. 2. Carnes, huevos y leguminosas secas. 3. Verduras y frutas. 4. Leche y productos lácteos. 5. Azúcares. 6. Grasas 14.

Este documento da cuenta de un estudio desarrollado con madres de niños en la edad preescolar de la comuna 9 de la ciudad de Armenia - Quindío, con el fin de identificar sus prácticas y conocimientos sobre la alimentación de sus hijos. Con la información obtenida se espera generar procesos educativos alrededor de la temática, que sean abordados con las madres, desde sus propios conocimientos, con estrategias que busquen mejorar la cultura de la alimentación en los niños en edad preescolar.

\section{MATERIALES Y MÉTODOS}

Este estudio se desarrolló durante el año 2015, fue 
abordado desde un enfoque cuantitativo, observacional, de tipo descriptivo, de corte transversal, avalado por los comités técnico científico y de bioética de la Corporación Universitaria Empresarial Alexander von Humboldt.

Se aplicó muestreo no probabilístico teniendo en cuenta criterios de inclusión y de exclusión: madres biológicas o adoptivas de niños entre 3 y 6 años, residentes de la comuna 9 de la ciudad de Armenia, cuidadoras principales de sus hijos, y que le dedicaran al cuidado de sus hijos por lo menos media jornada diaria.

La muestra estuvo constituida por 50 madres de niños en edad preescolar (entre 3 y 6 años) de la comuna 9 de la ciudad de Armenia, Quindío en el año 2015, especialmente en los barrios: La Nueva Libertad, El Granada y La Arboleda.

$\mathrm{Al}$ instrumento diseñado por los investigadores, se le aplicó prueba piloto con 10 madres de niños preescolares, teniendo en cuenta los criterios de inclusión y exclusión. El proceso se realizó con el fin de ajustar el mismo, según las observaciones de las participantes. Dichas mujeres no hicieron parte de los sujetos de estudio.

Las mujeres fueron visitadas en sus hogares, por los investigadores, quienes diseñaron y aplicaron una encuesta guiada tipo CAP15, compuesta por 20 preguntas, que permitió evaluar los conocimientos, las actitudes y prácticas de las madres frente a la alimentación de sus hijos en edad preescolar. Previa firma del consentimiento informado.

Con los datos obtenidos se construyó base de datos y el análisis univariado de la información se realizó mediante la utilización del software Microsoft Excel en su versión 2010.

\section{RESULTADOS}

\section{a. Variables sociales y demográficas}

El grupo de participantes estuvo conformado por 50 mujeres, madres de niños en edad preescolar y que residían en la comuna Nro. 9 de la ciudad de Armenia, Quindío.

Las edades del grupo de madres Estuvieron compren- didas entre los 18 y 54 años, los grupos más representativos lo constituyeron, aquel entre los 30 y 35 años, con un $32 \%$ y entre los 18 y 23 años con un $30 \%$. El promedio de edad fue de 30,7 años.

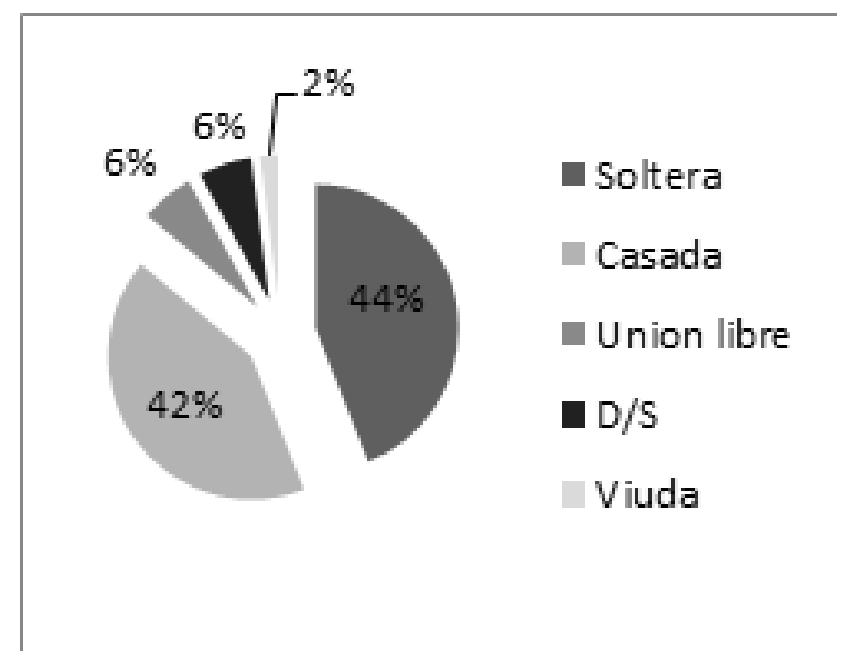

Gráfico 1. Estado civil de las madres

Con respecto al estado civil, se evidenció que la mayoría de las madres conviven con sus parejas, en esta clasificación se incluyen las parejas casadas y en unión libre, representada con un 48\%, seguido de las mujeres solteras que están representadas con un $44 \%$. Solo el 2\% de las mujeres del estudio presenta condición de viudez (ver gráfico 1 ).

Al indagar sobre el número de hijos, un $46 \%$ de las madres manifestó tener solo uno, seguidas de aquellas que tenían 2 hijos (28\%), 2\% de los encuestados manifestó tener 5 hijos

Las edades de los hijos de las mujeres encuestadas oscilaron entre los 4 meses y los 33 años.

En relación al nivel educativo, se evidenció que un $38 \%$ de las madres había alcanzado la secundaria completa, un $24 \%$ la secundaria incompleta. Un $6 \%$ había logrado nivel universitario.

Al indagar sobre la actividad laboral, la mayoría de las mujeres (52\%) expresaron ser amas de casa y un $40 \%$ ocupación laboral.

En relación con los ingresos económicos de la población estudiada, se encontró que un 52\% recibían un salario mínimo mensual legal vigente, un 30\% menos de un salario mínimo legal vigente y tan solo un $18 \%$ recibía más de un salario mínimo legal vigente, 
al mes.

El estrato socioeconómico de las madres, se puede evidenciar en la gráfica 2, donde predomina el nivel bajo.

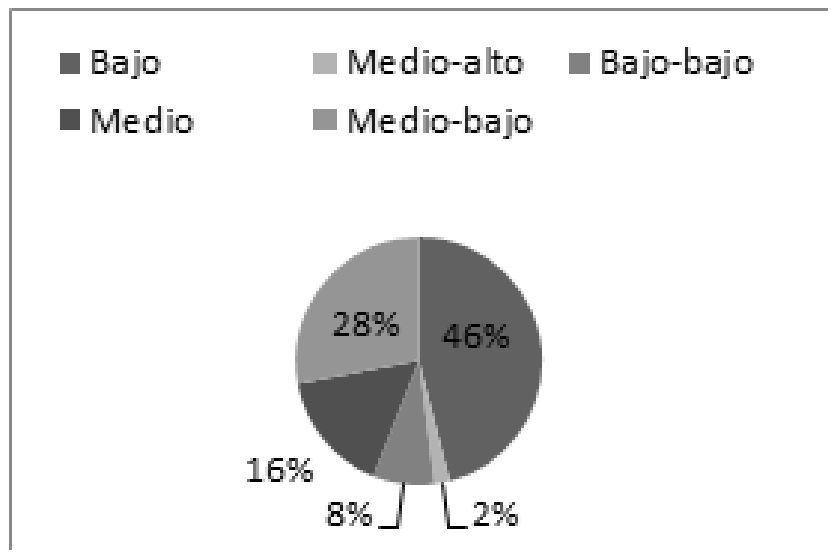

Gráfico 2. Nivel social de las madres

\section{b. Conocimientos}

La información obtenida acerca de los conocimientos de las madres frente a la alimentación de sus hijos, se encontró que un $44 \%$ consideraba que es conveniente brindar a los preescolares, seis porciones de alimentos al día, un 30\% refirieron que es conveniente brindar cinco porciones y tan solo $2 \%$ expresó que lo correcto era brindar siete porciones de alimentos al niño en edad preescolar.

Tabla 1. Grupos de alimentos que deben ser brindados con mayor frecuencia a los preescolares

\begin{tabular}{|l|c|}
\hline \multicolumn{1}{|c|}{ Grupos alimentos } & \% consumo diario \\
\hline $\begin{array}{l}\text { Cereales, tubérculos, raíces y } \\
\text { plátanos }\end{array}$ & $80 \%$ \\
\hline Frutas y verduras & $70 \%$ \\
\hline Lácteos & $50 \%$ \\
\hline Carnes y huevos & $76 \%$ \\
\hline Grasas & $10 \%$ \\
\hline Azúcares & $10 \%$ \\
\hline
\end{tabular}

Las madres que hicieron parte de la muestra de estudio expresaron, en relación con los grupos de alimentos indispensables para una adecuada alimentación de sus hijos, los constituyen granos, verduras, frutas, carnes y huevos, como los más importantes (ver tabla 1).

Cocinar (80\%) o asar (70\%), fueron las 2 formas con- sideradas como ideales, para preparar los alimentos de los preescolares según sus madres. Freír en aceite (14\%), freír en agua (12\%) y al vapor $(10 \%)$ fueron las opciones menos convenientes para los niños.

\section{c. Actitudes}

En cuanto a las actitudes de las madres frente a la alimentación de sus hijos, se identificó que más de la mitad tenían una actitud indiferente reflejada en un $66 \%$, solo el $2 \%$ presento una actitud inadecuada y $32 \%$ adecuada. (Ver gráfico 3 ).

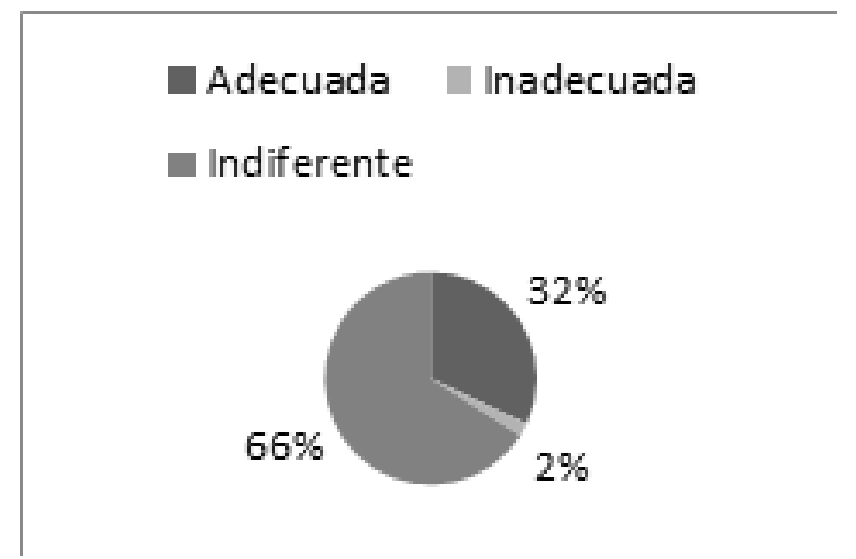

Gráfico 3. Actitudes de las madres frente a la alimentación del preescolar

\section{d. Prácticas}

Las prácticas de las madres sobre la alimentación de sus hijos preescolares, se indagaron a partir de los alimentos brindados en las 3 "comidas" principales: desayuno, almuerzo y comida.

Los hallazgos mostraron que un $22 \%$ de los niños toman biberón de 2 y 5 veces al día. Un $6 \%$ aún recibía leche materna.

$\mathrm{Al}$ indagar por el número de platos o de porciones de comida al día, se mostró que un $44 \%$ servía 3 platos, que un $26 \%$ ofrecía 5 platos, $12 \%$ ofrecía 4 platos, $10 \%$ ofrecía 3 platos, $6 \%$ ofrecía 8 platos y el $2 \%$ ofrecía 7 raciones de comida al día.

Con respecto a la porción de alimentos brindados en una comida, el 64\% de las madres aseguraban ofrecer un plato completo, un $20 \%$ brindaban medio plato, un $14 \%$ brindaban dos platos y tan solo un $2 \%$ contesto ninguna de las anteriores. 
En cuanto a la preparación de los alimentos un $46 \%$ de las madres eligieron cocinado, un $21 \%$ los asados y un $19 \%$ no respondieron a la pregunta.

Los tipos de alimentos brindados a los preescolares, en cada una de las comidas principales, se presentan en la tabla 2:

Tabla 2. Porcentaje de alimentos brindados a los preescolares en cada una de las comidas principales:

\begin{tabular}{|c|c|c|c|c|}
\hline Grupos Alimentos & Alimentos & Desayuno & Almuerzo & Comida \\
\hline \multirow{10}{*}{$\begin{array}{c}\text { Cereales } \\
\text { Tubérculos, raíces } \\
\text { y plátanos } \\
\text { (grupol) }\end{array}$} & Arroz & $7 \%$ & $46 \%$ & $37 \%$ \\
\hline & Pan & $36 \%$ & - & $4 \%$ \\
\hline & Galletas & $21 \%$ & $2 \%$ & $4 \%$ \\
\hline & Arepa & $23 \%$ & - & $12 \%$ \\
\hline & $\begin{array}{l}\text { Cereales } \\
\text { comerciales }\end{array}$ & $34 \%$ & - & $14 \%$ \\
\hline & Colada & $2 \%$ & - & - \\
\hline & Sopas y cremas & - & $45 \%$ & $36 \%$ \\
\hline & Papa & - & - & - \\
\hline & Plátano & - & $17 \%$ & $7 \%$ \\
\hline & No recibe & - & - & - \\
\hline \multirow{4}{*}{$\begin{array}{l}\text { Verduras y frutas } \\
\text { (grupo 2) }\end{array}$} & Jugos & $6 \%$ & $36 \%$ & $12 \%$ \\
\hline & Fruta entera & $6 \%$ & $17 \%$ & $9 \%$ \\
\hline & Verduras & - & $29 \%$ & $11 \%$ \\
\hline & No recibe & $88 \%$ & $18 \%$ & $68 \%$ \\
\hline \multirow{3}{*}{$\begin{array}{c}\text { Leche y productos } \\
\text { lácteos } \\
\text { (grupo 3) }\end{array}$} & Leche & $11 \%$ & - & $2 \%$ \\
\hline & Queso & $11 \%$ & - & - \\
\hline & No recibe & $78 \%$ & $100 \%$ & $98 \%$ \\
\hline \multirow{6}{*}{$\begin{array}{c}\text { Carnes, huevos y } \\
\text { Leguminosas secas } \\
\text { (grupo 4) }\end{array}$} & Carne & $2 \%$ & $40 \%$ & $28 \%$ \\
\hline & Pescado & - & - & $2 \%$ \\
\hline & Huevos & $28 \%$ & $4 \%$ & $14 \%$ \\
\hline & Granos & - & $17 \%$ & $14 \%$ \\
\hline & Carnes frias & $4 \%$ & - & $2 \%$ \\
\hline & No recibe & $66 \%$ & $39 \%$ & $40 \%$ \\
\hline $\begin{array}{l}\text { Azúcares } \\
\text { (Grupo 5) }\end{array}$ & Agua de panela & $12 \%$ & - & $12 \%$ \\
\hline \multirow{2}{*}{$\begin{array}{l}\text { Grasas } \\
\text { (grupo 6) }\end{array}$} & $\begin{array}{ll}\begin{array}{l}\text { Bebida } \\
\text { chocolate }\end{array} & \text { de }\end{array}$ & $35 \%$ & - & $12 \%$ \\
\hline & No recibe & $53 \%$ & $100 \%$ & $88 \%$ \\
\hline
\end{tabular}

En el cuadro anterior se relaciona cada uno de los grupos de alimentos que son brindados a diario a los preescolares y el porcentaje de consumo de los mismos, según lo reportado por sus madres. Es de resaltar que el grupo de "cereales tubérculos, raíces y plátanos" fue el brindado con mayor frecuencia durante el día, evidenciándose inclusive la combinación de varios alimentos de este grupo en el mismo plato.

En relación a los demás grupos de alimentos se identificó un bajo consumo, en su orden, el grupo de leches y productos lácteos, el grupo de frutas y verduras y el grupo de carnes huevos y leguminosas secas, los cuales deben ser brindados en mayor cantidad, según las recomendaciones del "Plato de la familia colombiana"14.
Es de resaltar que el consumo de grasas y azucares es limitado. Durante la encuesta no se indago sobre los refrigerios, dulces y "mecatos" brindados al niño diariamente.

El plato saludable de la familia colombiana14 recomienda consumir seis grupos de alimentos en el plato, con mayor consumo del grupo 1 donde se encuentran los cereales, tubérculos y hortalizas y limitar el consumo de los grupos 5 y 6 grasas y azucares.

\section{DISCUSIÓN}

Los hallazgos obtenidos dentro del presente estudio son preocupantes. Se evidencia que las madres tienen 
un amplio y apropiado conocimiento frente a los hábitos alimenticios de los preescolares, sin embargo, se identifica una actitud de indiferencia y de prácticas deficientes y poco apropiadas para el menor.

Los conocimientos que tienen las madres acerca de los alimentos que les brindan diariamente a sus hijos, son de vital importancia ya que le permitirían a los preescolares adquirir todos los nutrientes necesarios para su desarrollo físico y mental. Del mismo modo, permitirían que los niños iniciaran la adquisición de hábitos que gradualmente incorporarán en sus prácticas alimenticias. En este sentido, las madres encuestadas demostraron conocimientos amplios y adecuados con respecto a los alimentos, la frecuencia de su consumo y preparación más adecuada, para los preescolares.

En contraste, estudios realizados en población similar, como uno reportado en Ecuador en el año 201216 muestra que las madres presentaban un conocimiento deficiente acerca de la adecuada alimentación que debe ser brindada a los niños en edad preescolar.

Sin embargo, en un estudio realizado con madres de preescolares en una institución educativa de Lima, Perú en el año 200617, se pudo evidenciar, a partir de pruebas estadísticamente significativas, que el nivel "medio-bajo" de conocimientos (74\%) de las participantes, sobre el contenido ideal de la lonchera de los preescolares, se relacionaba con unas prácticas inadecuadas $(76,7 \%)$.

En este mismo sentido, un estudio tipo CAP realizado con madres de niños menores de 1 año, sobre la alimentación de los mismos, (Lima, Perú 2011)18, mostró relación entre los conocimientos positivos $(55 \%)$ y las prácticas positivas $(61 \%)$ de las madres hacia la alimentación de sus hijos.

Barrera LF, Herrera GM, Ospina JM (2014), reportaron que se encontró déficit de conocimientos y frecuencia de consumo inadecuadas como factores de riesgo posiblemente asociados a la desnutrición crónica; creencias en nutrición no favorables, frecuencia de consumo inadecuada de alimentos lácteos, son factores de riesgo posiblemente asociados a desnutrición aguda.

La actitud que las madres tengan respecto a la alimentación de los niños es importante para transmitir a ellos la seguridad y confianza que necesitan para saber que ese alimento que están consumiendo o van a consumir, traerá beneficios a su vida y contribuirá en su crecimiento y desarrollo. En las madres encuestadas de la comuna nueve de Armenia-Quindío se identificó que más de la mitad de ellas tiene una actitud indiferente hacia la alimentación de sus hijos en edad preescolar, lo que quiere decir que a pesar de tener conocimientos adecuados acerca del tipo de alimentos que deben brindarle a los niños, su actitud no contribuye a que el preescolar adquiera de manera segura, los hábitos correctos para su alimentación.

Este tema es abordado en el libro blanco19 de la nutrición infantil (España), en el cual se hace mención acerca de la inadecuada actitud de los padres como una amenaza para el desarrollo de los preescolares, debido a que esto induce al desarrollo de hábitos poco saludables en la alimentación de los niños, ya que el comportamiento que tienen sus padres frente a ellos es tomado como una conducta a seguir.

En torno a la actitud de los padres, el estudio de Parada Rico (2011)11, ya citado en este texto, advierte que se identifica una actitud pasiva de vigilancia y cuidado de parte de los padres en el crecimiento del niño/a, refiriendo que los menores son de padres de baja talla por lo cual también deben poseer sus mismas características físicas de éstos, sin preocuparse por las deficiencias del menor en relación con los controles de crecimiento y desarrollo, procesos en los cuales la alimentación juega un papel vital.

Con respecto a las prácticas, las madres proporcionan una adecuada cantidad y frecuencia de los alimentos a los preescolares. Sin embargo, se observó que las mujeres brindan pocas frutas, verduras, lácteos y carnes a sus hijos durante el día, alimentos que son importantes porque brindan los macro y micro nutrientes necesarios para el adecuado funcionamiento y desarrollo del organismo del menor. En este sentido, un estudio realizado en México (2016)20, sobre pautas alimentarias para la incorporación de la dieta familiar, menciona la disminución del consumo de frutas y verduras desde el momento en que los niños se encuentran en dicha inclusión.

En estudio de Delgado Pérez (2016)21, dentro de los resultados se reporta que las madres de preescolares, en relación con las estrategias utilizadas para para alimentar a sus hijos, aplicaron tanto estrategias positi- 
vas en forma responsiva, como negativas, en forma de presión, chantaje para que sus hijos consuman la cantidad que ellas consideraban adecuada.

Por otro lado, se evidenció un alto consumo de carbohidratos, que si no se hace de forma controlada puede generar un amento en el peso de los niños.

Todo esto permite identificar lo importante que es desde edades tempranas brindar a los niños alimentos saludables e incentivarlos a consumir verduras y frutas, para que este hábito se fortalezca en ellos a medida que transcurren los años, evitando que en un futuro padezcan las consecuencias de una alimentación inadecuada tales como obesidad y malnutrición.

Un estudio realizado en Perú (2014)22, relaciona las prácticas alimenticias con el estado de ánimo del niño y la forma en que se le brindan los alimentos, comenta que cuando las personas encargadas de ofrecerle la alimentación al niño, tienen grandes expectativas sobre su comportamiento a la hora de darle el alimento, al no ser controlado el menor, el cuidador reacciona de manera agresiva, creando temor y ansiedad en el pequeño, lo que conlleva a retardar el flujo del jugo gástrico e inhibir la ingestión, afectando las practicas alimenticias saludables.

Las relaciones nutrición-salud se han ido estableciendo cada vez con mayores evidencias. Los estilos de vida y los hábitos alimentarios de las sociedades trazan en buena parte su salud y previenen y promueven la aparición de enfermedades crónicas durante la vida, sin embargo, estos trastornos que se conforman en la vida adulta, comienzan a desarrollarse en las primeras etapas de la vida, por eso resulta del mayor interés examinar cuáles son las necesidades y recomendaciones nutricionales durante la infancia y la adolescencia23.

En relación a las características socioeconómicas reportadas por las madres encuestadas, al menos el 50\% de las mismas recibían un salario mínimo. En este sentido, no se hallan estudios que puedan ser comparables con los datos reportados en la presente investigación.

Como ya se ha expresado, a pesar de que no se ha explorado en profundidad la incidencia del ingreso económico bruto de la familia en las prácticas alimentarias, sí se puede inferir, basados en los preca- rios ingresos económicos reportados por las madres, que éste aspecto incide directamente en la cantidad y calidad de la alimentación a la que acceden los niños. Estudios realizados en Cataluña, Canarias y Granada por Capdevila F, Llop D, Guillén N, Luque V, Pérez S, Sellés V, et al. (2012)24 y González Jiménez E, Aguilar Cordero MJ, García García CJ, García López P, Álvarez Ferre J, Padilla López CA, et al. (2014)25, así lo confirman, estos autores encontraron menor frecuencia de consumo de verduras y frutas en niños de posición socioeconómica baja.

Pabayo R, Spence JC, Cutumisu N, Casey L, Storey K.(2012)26 realizaron un estudio en Canadá en niños de 4 y 5 años el cual reveló mayor frecuencia de consumo de refrescos azucarados entre aquellos de clase social baja, y otro estudio en adolescentes de EE. UU. Llevado a cabo por Cutler GJ, Flood A, Hannan P, Neumark-Sztainer D. (2012)27 dejó en evidencia una clara relación entre el consumo de comida rápida con factores sociodemográficos.

Los datos reportados de la literatura internacional podrían ayudar a comprender hasta cierto punto ciertas características de la alimentación de los hogares caracterizados por bajos niveles de ingresos económicos y bajo estrato socioeconómico de este estudio, pues se ha encontrado que el $52 \%$ de los sujetos que constituyeron la muestra escasamente perciben un ingreso mensual de un salario mínimo y el 30\% ni siquiera alcanza este monto, lo cual impide que estas familias puedan acceder a una canasta familiar básica, es decir, el $82 \%$ de los estudiados presentan problemas económicos, solo un $18 \%$ del total de la muestra tiene un ingresos superior a un salario mínimo, lo cual tampoco significa que puedan acceder a los grupos de alimentos más costosos, que en nuestro país están representados por un lado por proteínas de origen animal como carnes, leche y sus derivados y por otro lado algunas frutas y verduras.

Otros aspectos derivados de las características sociodemográficas, según algunos estudios tienen repercusiones en las prácticas alimentarias promovidos por las madres hacia sus hijos. Dynesen AW, Haraldsdottir J, Hola L, Astrup A. (2003) 28 y Roos EB, Hirvonen T, Mikkila V, Karvonen S, Rimpela S. (2001)29 en Finlandia y Dinamarca respectivamente, observaron que los niños cuya madre tenía nivel de estudios bajo mostraban un consumo elevado de snacks y un consumo inferior al deseado de vegetales y frutas. 
Una cierta actitud de indiferencia por parte de las madres en estudio frente a la alimentación de sus hijos, podría también explicarse por su falta de conocimiento y preparación en el plano académico.

Probablemente la educación y la posición socioeconómica de los padres influye en los hábitos alimentarios, facilitando o restringiendo la comprensión de la información nutricional y el cumplimiento de las recomendaciones nutricionales. Para algunos autores existe cierto grado de controversia sobre si el nivel de formación de los padres, en especial el de la madre, influye en la nutrición de los hijos durante toda la etapa infantil y la adolescencia, o si su influencia se vería limitada hasta los 10 años de edad.

Al revisar el nivel escolar de las madres, el presente estudio reporta un nivel técnico o universitario en un $6 \%$ de la población y un $38 \%$ con secundaria completa. En estudios similares en Lima, Perú 200617, las madres reportaron un $45,3 \%$ con secundaria completa y un $22,7 \%$ con estudios superiores, sin embargo, su nivel de conocimiento y práctica fue deficiente, contrastando ambos datos con el nivel de nutrición de los niños. En el presente estudio, a pesar de tener un nivel inferior de escolaridad, los conocimientos de las madres, fueron altos, aunque las prácticas, deficientes.

Del mismo modo, el estudio de Perú17 reportó un $78 \%$ de madres amas de casa y un $22 \%$ de madres dedicadas a otras labores, estos datos no fueron relacionados con el nivel de conocimientos y prácticas, los cuales se reportaron como bajos o deficientes. En contraste, el presente estudio reportó un 52\% de madres amas de casa y un $40 \%$ de madres que laboraban; los conocimientos de las mismas, se reportó como adecuado, aunque las prácticas, como deficientes.

\section{CONCLUSIONES}

En relación a los hallazgos demográficos y sociales, se puede evidenciar que las madres participantes, eran jóvenes con 2 o 3 hijos, amas de casa, pertenecientes a estratos económicos bajos y con pocos ingresos económicos. La limitación de estudios similares dentro del país, impide que estos datos puedan ser contrastados.
Aunque las madres tengan adecuados conocimientos frente a la alimentación de sus hijos las actitudes son indiferentes y las prácticas deficientes, esto puede deberse a las características sociales de las mismas, a pesar de ser una variable no indagada, es probable que las condiciones socioeconómicas estén incidiendo negativamente en las prácticas alimentarias de las madres hacia sus hijos, lo que pueden limitar el consumo adecuado o necesario que cubran los requerimientos diarios de alimentos en los preescolares.

Se hace necesario realizar estudios similares en población más amplia, en otras comunas de la ciudad y con otras características sociales y demográficas, que permitan realizar generalización de los datos y grupos comparativos, que puedan acercar la información a relaciones causales entre algunos aspectos sociales y económicos, con los conocimientos y las prácticas de las madres, hacia hábitos de sus hijos preescolares.

\section{LIMITACIONES}

Las limitaciones del presente estudio se dan en dos aspectos principales. El primero tiene que ver con el tipo de muestreo, no probabilístico, lo que impide realizar generalización de los hallazgos.

El segundo, se relaciona con el instrumento utilizado, el cual fue sometido a prueba piloto, con el propósito de dotarlo de validez de contenido, al asegurar la integridad de las preguntas y la comprensión de las mismas por parte de los sujetos de estudio. No se puede asegurar una validez total, dado que no se realizaron pruebas de validez de criterio, debido a que no se pudo acceder a instrumentos estandarizados que pretendieran medir lo mismo, por lo que se indaga en este estudio.

Por otro lado constituye una limitación el hecho que el estudio solo cubrió la zona urbana, por lo tanto los resultados no son generalizables a las zonas rurales de la ciudad.

Finalmente, los reportes de consumo que tienen que ver directamente con las prácticas no obedecen a un registro y seguimiento de las mismas sino que han sido reportados por las madres por medio de cuestionario. 


\section{BIBLIOGRAFÍA}

1. Franco, S. M. (2010). Aportes de la sociología al estudio de la alimentación familiar. Revisa Luna Azul, 31(10).

2. Osses, S, Macías, C, Castaño, S, López, A y Gómez del Castillo, D. (2014). Representaciones sociales que orientan prácticas de cuidado de la salud en la Primera Infancia: Una aproximación al estado del arte. Artículo en prensa.

3. Contigo salud vivir mejor: Alimentación de los niños de 2 a 5 años. [citado el 15 de marzo 2014]. Disponible en URL: http://www.contigosalud.com/alimentacion-en-ninos-2-a-5-anos

4. Turiani M, Pamplona Tonete V L, Komura Hoga L A, Chávez Álvarez R E. El cuidado del niño: Representaciones y experiencias de la madre adolescente de bajos recursos. Granada. Index Enferm. abr.-jun. 2009; 18(2).

5. Barrera Sánchez LF, Herrera Amaya M, Ospina Día JM. Intervención educativa para modificación de conocimientos, actitudes y prácticas sobre nutrición en Boyacá, Colombia. Cuidarte. 2014; 5 (2).

6. Organización Mundial de la Salud OMS. Temas de salud: Nutrición. [internet]. [Consultado 2016 Dic. 17]. Disponible en URL: http://www.who.int/features/factfiles/nutrition/es/

7. Organización Mundial de la Salud. (2010). Population based prevention strategies for childhood obesity: report of a WHO forum and technical meeting. Geneva: WHO.

8. UNICEF/WHO/World Bank. (2013). Joint Child Malnutrition Estimates.

9. Profamilia, ENDS 2010. Lactancia Materna y Estado Nutricional.

10. Sáenz M. y Camacho Á. Prácticas de Lactancia Materna y Alimentación Complementaria en un Jardín Infantil de Bogotá. Revista de Salud Pública 2007 ; 9 (4) ): 587 - 594

11. Parada R DA. Conocimientos actitudes y prácticas del cuidado del menor de cinco años. Revista Ciencia y Cuidado. 2011; 8(1):29-39.

12. Ministerio de Salud y de Protección Social. Indicadores básicos 2013: situación de salud de Colombia. Colombia. 2014.

13. Secretaria de Salud Departamental del Quindío. Análisis de situación de salud en el modelo de los determinantes sociales de la salud, departamento del Quindío 2016. Armenia, Colombia 2016.

14. Sociedad Colombiana de Cardiología y Cirugía Cardiovascular. Fundación Colombiana del Corazón: Corazones sanos - Guía para que la familia aprenda cómo alimentarse para tener un corazón sano. [citado el 10 de marzo del 2015]. Disponible en URL: https://www.youtube.com/watch?v=6Rcrgb8ke5uk

15. Save the children. Encuestas de Conocimientos, Actitudes y Prácticas en el ámbito de la Protección de la Infancia [internet]. [Consultado 2015 Febr 27]. Disponible en URL: http://resourcecentre.savethechildren.se/sites/default/files/documents/kap_report_sp_hi-res.pdf

16. Asencio borbor $1 \mathrm{M}$, Muñoz Reyes KJ. Influencia de los factores socioeconómicos y culturales en el estado nutricional de los preescolares según su alimentación en las guarderías "san pablito" y "mis primeros pasos" comuna san pablo. Santa Elena. 2011-2012. Licenciado en enfermería. La libertad: universidad estatal península de santa Elena; 2012.

17. Requena Raygada L. Conocimiento y prácticas que tienen las madres sobre el contenido de la lonchera y su relación con el estado nutricional del preescolar de la Institución educativa Nr. 524 Nuestra Señora de la Esperanza. Lima, Perú. 2006.

18. Galindo Bazalar D. Conocimientos y prácticas de las madres de niños de 6 a 12 meses sobre alimentación complementaria en el Centro de Salud "Nueva Esperanza". Universidad Nacional Mayor de San Marcos. Lima, Perú. 2011.

19. Rivero M, Moreno L, Dalmau J, Moreno J, Aliaga A, García A, Varela G, Ávila J. LIBRO blanco de

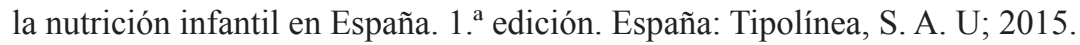

20. Martínez R, Alvarado G, Sánchez M, Blanco L, Sánchez M, Blázquez J, Méndez S. Estudio de las pautas alimentarias para la introducción de alimentos complementarios y su diversidad a través de la incorporación a la dieta familiar. Revista de Especialidades Médico-Quirúrgicas 2016;15(3):114-124. Disponible en: http://www.medigraphic.com/pdfs/quirurgicas/rmq-2010/rmq103d.pdf

21. Delgado D. Estrategias usadas para alimentar a niños preescolares por madres de una zona urbana marginal de Lima, Perú. Rev Peru Med Exp Salud Publica. 2016;33(3):507-12.

22. Mamani E. relación las practicas alimentarias de las madres con el estado nutricional de los niños y niñas en la etapa preescolar y escolar de aldeas infantiles sos juliaca, septiembre - diciembre del 2013.peru. (internet). 2014 [citado 15 abril 2016]. Disponible en: http://es.slideshare.net/edithania1/ practicas-alimentarias-y-estado-nutricional-fin 
23. Suárez et al. Conocimientos, actitudes y prácticas sobre alimentación y nutrición de adolescentes de educación media. MedULA 23: 83-91. 2014.

24. Capdevila F, Llop D, Guillén N, Luque V, Pérez S, Sellés V, et al. Consumo, hábitos alimentarios y estado nutricional de la población de Reus $(\mathrm{X})$ : evolución de la ingestión alimentaria y de la contribución de los macronutrientes al aporte energético (1983-1993) según edad y sexo. Med Clin (Barc). 2000;1:7---14.

25. González Jiménez E, Aguilar Cordero MJ, García García CJ, García López P, Álvarez Ferre J, Padilla López CA, et al. Influence of family environment of the development of obesity and overweight in a population of school children in Granada (Spain). Nutr Hosp. 2012;27:177---84

26. Pabayo R, Spence JC, Cutumisu N, Casey L, Storey K. Sociodemographic, behavioural and environmental correlates of sweetened beverage consumption among pre-school children. Public Health Nutr. 2012;15:1338---46.

27. Cutler GJ, Flood A, Hannan P, Neumark-Sztainer D. Multiple sociodemographic and socioenvironmental characteristics are correlated with major patterns of dietary intake in adolescents. J Am Diet Assoc. 2011;111:230---40.

28. Dynesen AW, Haraldsdottir J, Hola L, Astrup A. Sociodemographic differences in dietary habits described by food frequency questions-results from Denmark. Eur J Clin Nutr. 2003;57:1586---97.

29. Roos EB, Hirvonen T, Mikkila V, Karvonen S, Rimpela S. Household educational level as determinant of consumption of raw vegetables among male and female adolescent. Prev Med. 2001;33:282---91 